\title{
Forensic Study of Indian Toxicological Plants as Botanical Weapon (BW): A Review
}

Balvant S. Khajja*, Mukesh Sharma, Rajveer Singh and Girish K. Mathur

State Forensic Science Laboratory, Jaipur, Rajasthan, India

\begin{abstract}
Poisons, those silent weapons capable of destroying life mysteriously, secretly and without violence, have ever had a peculiar fascination for mankind. The plant originated weapons or "BOTANICAL WEAPONS (BW)" were also used by criminals in burglary, rape and murder cases. The Botanical weapons are may be the part of the toxic and poisonous plants or may be the toxic constituents of plants.

In India, there are so many cases were available where criminal by mixing it in food material or contact of the irritating toxic parts of the plants to victim's body in the buses/trains. Poisonous plants are those biological weapons which cause serious problems or even death occur.

These weapons are first choice of professional poisoners in toxi-crime because they are easily available and having no cost. They have played so large a part, in romance as well as in crime, that the subject is one which claims the attention of even student of human nature. Through this article authors have studied some toxic and poisonous plants of India, which are generally used by the criminals to commit the crimes. In this paper, all the toxicological substances of these plants are also mentioned, which provide a prefect database for the forensic toxicologists.
\end{abstract}

Keywords: Botanical Weapons; Toxic Plants; Toxi-crime; Bioterrorism

\section{Introduction}

A poison is a substance which, when administered, inhaled or ingested, is capable of acting deleteriously on the human body. Thus, there are really no limits, between a medicine and a poison, for a medicine in a toxic dose is a poison and a poison in a small dose may be a medicine means, it depends on dose/quantity only. In law, the real difference between a medicine and a poison is the intent with which it is given. If the substance is given with the intention to save life, it is a medicine but if it is given with the intention to cause bodily harm, it is a poison [1]. It is fact that virtually any substances can be harmful at high concentration- as Paracelsus (1493-1541), the father of toxicology said in the sixteenth century, "Everything is poison, there is poison in everything, only the dose makes a thing not a poison" [2]. In the context of biology, poisons are substances that can cause disturbances to organisms. Throughout human history, intentional application of poison has been used as a method of assassination, murder, suicide, and execution. Poison includes both naturally produced compounds and chemicals manufactured by humans. Natural poisons are produced by species of bacteria, fungi, protists, plants and animals. Poisonous plants are those which cause serious problems or even death occur, if a small quantity of its stem, leaves, seeds, fruits and roots are ingested [3]. Some other plants are normally harmless but they may become toxic if preparative from them are taken in excess in strong doses or for along period of time as suggested by Qureshi et al. [4].

Poisoned weapons were used in ancient India, and war tactics in ancient India have references to poison. A verse in Sanskrit reads "Jalam visravayet sarmavamavisravyam ca dusayet," which translates to "Waters of wells were to be mixed with poison and thus polluted."Chanakya (c. 350-283 BC), also known as Kautilya, was adviser and prime minister to the first Maurya Emperor Chandragupta (c. 340-293 BC). Kautilya suggested employing means such as seduction, secret use of weapons, and poison for political gain [5]. Susruta Samhita has described several modes of poisoning in ancient
India. He has explained how the poisons are mixed with food, drink, honey, and snuff or sprinkled over cloths, beds, couches, shoes, garlands and jewellary, saddles of horses, etc. Therefore, it may be concluded that these practices were in vogue in his time [6]. Gradually, there arose a class of "professional poisoners" who could ingeniously mask the bitter taste or strange odours of the poisons with sweet tasting and pleasant substances [1].

The incidence of poisoning in India is among the highest in the world, and it is estimated that more than 50,000 people die every year from toxic exposure [7]. The causes of poisoning are many - civilian and industrial, accidental and deliberate. The commonest agents in India appear to be pesticides (organophosphates, carbamates, chlorinated hydrocarbons, and pyrethroids), sedative drugs, chemicals (corrosive acids and copper sulfate), alcohols, plant toxins (datura, oleander, strychnos, and gastro-intestinal irritants such as castor, croton, calotropis, etc.), and household poisons (mostly cleaning agents). [8]. Poisonous plants of India have been described by few workers [9-10]. A lot of work has been reported on toxicology of plants but no work has been done specially on poisonous plants study in terms of forensic context. In the present study, a review has been performed on almost poisonous plants of India about their fatal dose and fatal period. In this article, we reported basic details such as the botanical and family names, toxic parts of plant, chemical constituents and information about fatal dose and fatal period of the important plants.

*Corresponding author: Balvant S. Khajja, State Forensic Science Laboratory, Jaipur, Rajasthan, India, E-mail: balvantsingh@yahoo.co.in

Received September 20, 2011; Accepted November 20, 2011; Published November 23, 2011

Citation: Khajja BS, Sharma M, Singh R, Mathur GK (2011) Forensic Study of Indian Toxicological Plants as Botanical Weapon (BW): A Review. J Environment Analytic Toxicol 1:112. doi:10.4172/2161-0525.1000112

Copyright: @ 2011 Khajja BS, et al. This is an open-access article distributed unde the terms of the Creative Commons Attribution License, which permits unrestricted use, distribution, and reproduction in any medium, provided the original author and source are credited. 


\section{Poisoning in India}

There are more than 4000 species of medicinal plants growing as herbs, shrubs, and trees in India, many of which are poisonous when administered in large doses. The toxic principles belong to alkaloids, glycosieds, toxalbumins, resind, cannabinoides and polypeptides. Suicide in India, as poison can be easily obtained and many poisonous plants grow wild, eg.datura, oleanders, aconite, nux vomica, etc. Many Indians consider the taking of life by blood-shed a greater crime than poisoning, strangling etc. Accidental poisoning occurs from the use of philters or love potions and quack remedies containing poisonous drugs. The incidence of poisoning in India is among the highest in the world, and it is estimated that more than 50,000 people die every year from toxic exposure [3]. The causes of poisoning are many - civilian and industrial, accidental and deliberate. One recent study pertaining to poisoning statistics demonstrated more of such differences between northern and southern Indian states [11].

\section{Classification of Poison}

According to their mode of action, poisons are broadly classified in three groups, these three broad groups are sub-divided on the basis of their effect on the body, type of composition etc., which are available in the literature [1-3] and summarized as shown in Figure 1.

\section{Criminal Offences in Indian Panel Code (IPC)}

The administration of a poison is a criminal offence whenever (i) It is with intent to kill, (ii) with intent to cause serious injury, (iii) used recklessly even though there is no intent to kill, (iv) for stupefying to facilitate a crime, eg., robbery or rape, (v) to procure an abortion, (vi) to annoy the victim, (vii) to throw poison on another person with intention to injure him [11].

Now a day, in India mostly poisons are used for robbery and suicidal purposes. For example Datura is used by that sect of the thugs who poisoned wayfarers. Even today the poisoning and robbing of travelers was of frequent occurrence in India. By the judicious use of datura a whole household can be so drugged that the thieves can ransack the house at their ease. Datura has frequently been detected in the vomit of the victims of a midnight robbery. Red chilli powder is frequently used in the robbery or a confession of some guilt by introducing it into the nostrils, eyes, urethra, vagina, or rectum. Hyocyamus is used in war to control shell-shock. Poisoning is generally accidental though an overdose or rarely homicidal as in the crippen case. In forensic work,

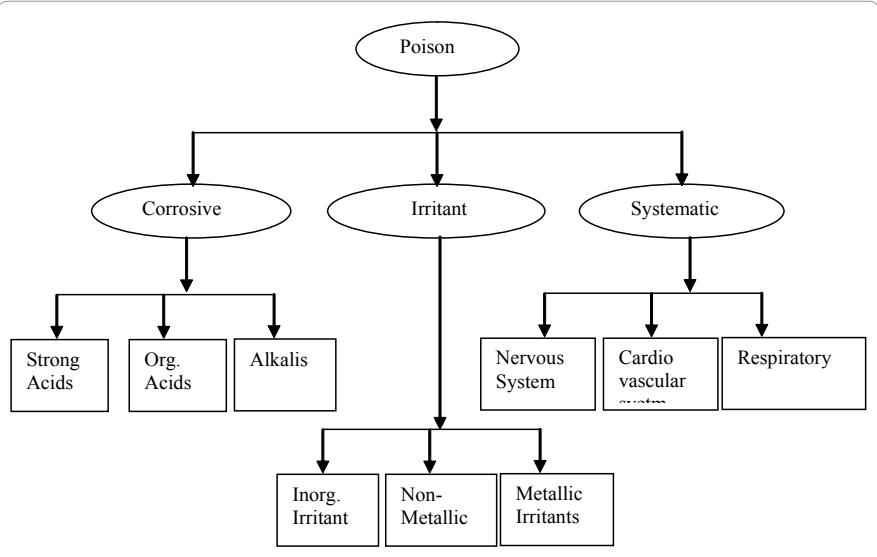

Figure1: Types of poison in clinical point of view. it can be used as a truth serum or lie detector. Hemlock was Athenian state-poison by which Socrates died.

\section{Characteristics of Ideal Poison}

The characters of an ideal homicidal/suicidal poison should be (i) cheap, (ii) easily available, (iii) colourless, odorless and tasteless, (iv) capable of being administered, either in food, drink or medicine, without producing any obvious change to prevent suspicion, (v) highly toxic (vi) capable of painless death (vii) signs and symptoms should resemble a natural disease, or the serious ill effects should be delayed sufficiently long for the accused to escape suspicion, (viii) must be rapidly destroyed or made undetectable in the body.

The number of factors, which are affecting the characteristics of poisoning any victim's as dose/quantity given to the victim, in which form (physical/chemical) the poison inject to the body, resistivity of body and condition of the victim (he/she might be drug addict/ in sleep or intoxication). Unless the poison is given in liquid form or small amount, the greater part of it may be lost by vomiting. However, the reorganization of poisoning during life is a matter of the first important, both from the point of view of the medical jurist and as a forensic crime scene investigator.

\section{Review}

It is essential to take cognizance of the fact that overuse or abuse of the medicinal constituents of plants can cause danger [9]. Plants containing glucosides, acids or alkaloids are used as medicines. Thus when taken in excess often have adverse effect. The latex, white or coloured sap found in families of Apocynaceae, Asclepiadaceae, Sapotaceae, Euphorbiaceae and Papaveraceae, if used in excess always act as poison. Plants of family Araceae have calcium carbonate oxalate crystals, which cause intense irritation of mouth and throat, as also swelling of throat and intestinal lining. This may cause suffocation or death.

Some plants containing orthophosphoric acids cause painful irritation and eruption if they came in contact with skin or mucous membrane. There are some plants or products like seeds of Annona squamosa L. and unripe pineapple when consumed induce abortion in pregnant women. The review on all toxic plants has been summarized in Table 1, this table provide a fundamental database for the forensic community which has been reviewed with the available literature [12 18], those who are working the field of forensic crime scene and as well as toxicologist as standard comparison during laboratory examination, which plants contains which type of constituents in it. Even plant toxins were quite low in incidence, which is because of the difficulty in testing for such toxins in the laboratory, as compared to chemicals.

\section{Conclusion}

In this paper, more than 50 poisonous plant species belonging to number of families are reported in the present paper. The poisonous parts of the majority of plants species were seeds, latex and root or root bark. Besides these poisonous parts of some plants were fruits, stem bark, tubers or bulbs and sometimes whole plant also. Some plants causes poisoning to both human beings as well as livestock populations, while some causes poisoning to human being only [19].

There are many plants which have no medicinal value and not used for the edible purpose but which are at times ingested through oversight, particularly by children. Many of these are responsible for poisoning in cattle. Some of the plants have been used for the poisoning purposes and for committing suicide. 
Citation: Khajja BS, Sharma M, Singh R, Mathur GK (2011) Forensic Study of Indian Toxicological Plants as Botanical Weapon (BW): A Review. J Environment Analytic Toxicol 1:112. doi:10.4172/2161-0525.1000112

Page 3 of 5

\begin{tabular}{|c|c|c|c|c|c|c|}
\hline Sr.No. & Name of Plant/Family & $\begin{array}{l}\text { Common Name/Hindi } \\
\text { Name }\end{array}$ & Toxic Parts & Toxic Constituents & Fatal Dose & Fatal period \\
\hline 1. & $\begin{array}{l}\text { Abrus precatorius } \\
\text { (Fabaceae) }\end{array}$ & $\begin{array}{l}\text { Rosary pea, Crab's eyes, } \\
\text { Gunchi (Hindi) }\end{array}$ & $\begin{array}{l}\text { Roots, seeds and } \\
\text { leaves }\end{array}$ & Abrin, Abrine and Abrasine & $\begin{array}{l}1 \text { - } 2 \text { seed or } \\
90-120 \mathrm{mg} / \mathrm{kg} \text { (Abrin) }\end{array}$ & $3-5$ days \\
\hline 2. & $\begin{array}{l}\text { Aconitum napellus } \\
\text { (Ranunculaceae) }\end{array}$ & $\begin{array}{l}\text { Indian aconite, } \\
\text { Monkshood and mitha } \\
\text { zahar (Hindi) }\end{array}$ & $\begin{array}{l}\text { All parts especially } \\
\text { Dried tuberous root }\end{array}$ & $\begin{array}{l}\text { Aconitine, Pseudo Aconite, } \\
\text { Indaconitine Bhikhaconitine, } \\
\text { Picraconitine, } \\
\text { and Aconine }\end{array}$ & $\begin{array}{l}1 \text { - } 2 \text { gram (root) } \\
1 \text { - } 2 \text { mg (Aconotine) }\end{array}$ & 2 to $6 \mathrm{~h}$ \\
\hline 3. & $\begin{array}{l}\text { Adenia palmata } \\
\text { (Passifloraceae) }\end{array}$ & & Fruit & $\begin{array}{l}\text { Toxalbumin (Cyanogenic } \\
\text { glycoside) and Emulsin (enzyme) }\end{array}$ & - & - \\
\hline 4. & $\begin{array}{l}\text { Aesculus hippocastanum } \\
\text { (Hippocastanaceae) }\end{array}$ & $\begin{array}{l}\text { Horse -chestnut, } \\
\text { conker }\end{array}$ & $\begin{array}{l}\text { All parts especially } \\
\text { seeds }\end{array}$ & Aescin and Aesculin & - & - \\
\hline 5. & $\begin{array}{l}\text { Alocasia macrorrhiza } \\
\text { (Araceae) }\end{array}$ & Giant taro, Elephant ear & All parts & $\begin{array}{l}\text { Calcium oxalate crystals } \\
\text { and toxic Proteins }\end{array}$ & $\begin{array}{l}1 / 30 \text { to } 1 / 15 \text { of } \\
\text { a grain }\end{array}$ & $15 \min$ \\
\hline 6. & $\begin{array}{l}\text { Anamirta cocculus } \\
\text { (Menispermaceae) }\end{array}$ & Indian berry or fish berry & Fresh fruit & $\begin{array}{l}\text { Picrotoxin and } \\
\text { Dihydro-picrotoxine }\end{array}$ & - & - \\
\hline 7. & $\begin{array}{l}\text { Antiaris toxicaria } \\
\text { (Moraceae) }\end{array}$ & Upas tree, Antiaris & Leaves and bark & a- Antiarin & $\begin{array}{l}\mathrm{LD}-0.116 \mathrm{mg} / \mathrm{kg} \text { i.v. } \alpha- \\
\text { antiarin }\end{array}$ & $2-3$ days \\
\hline 8. & $\begin{array}{l}\text { Argemone mexicana } \\
\text { (Papaveraceae) }\end{array}$ & $\begin{array}{l}\text { Argemone and Sial-kanta } \\
\text { (Hindi) }\end{array}$ & $\begin{array}{l}\text { All parts especially } \\
\text { seeds }\end{array}$ & $\begin{array}{l}\text { Berberine, Protopine, } \\
\text { Sanguinarine and } \\
\text { Dihydro-Sangunarine }\end{array}$ & - & - \\
\hline 9. & $\begin{array}{l}\text { Atropa belladonna } \\
\text { ( Solanaceae) }\end{array}$ & Deadly nightshade & All parts & $\begin{array}{l}\text { Atropine, Scopolamine, } \\
\text { Hyoscyamine, and } \\
\text { Belladonnine }\end{array}$ & $\begin{array}{l}120 \text { mg (atropine) } \\
30 \text { mg (hyoscine) }\end{array}$ & $24 \mathrm{~h}$ \\
\hline 10. & $\begin{array}{l}\text { Calotropis gigentea } \\
\text { (Apocyanaceae) }\end{array}$ & $\begin{array}{l}\text { Calotropis and madar, } \\
\text { akdo(Hindi) }\end{array}$ & Juice and roots & $\begin{array}{l}\text { Uscharin, Calotoxin, } \\
\text { Calactin and } \\
\text { Calotropin }\end{array}$ & $0.12 \mathrm{mg} / \mathrm{kg}$ calotropin & 12 to $24 \mathrm{~h}$ \\
\hline 11. & $\begin{array}{l}\text { Cannabis sativa } \\
\text { (Cannabinaceae) }\end{array}$ & Indian hemp Hashish & $\begin{array}{l}\text { Bhang- Dried } \\
\text { Leaves \& fruit } \\
\text { shoots } \\
\text { Majoon- Sweet } \\
\text { prepared with } \\
\text { bhang } \\
\text { Ganja- flowers top } \\
\text { of female plant } \\
\text { Charas- resin of } \\
\text { leaves and stems }\end{array}$ & $\begin{array}{l}\text { Cannabin, Cannabinon } \\
\text { and Cannabinol }\end{array}$ & $\begin{array}{l}10 \text { gm/kg b.wt.-bhang, } \\
8 \text { gm-ganja, } \\
2 \text { gram-charas }\end{array}$ & 5 - 8 Days \\
\hline 12. & $\begin{array}{l}\text { Capsicum annum } \\
\text { (Solanaceae) }\end{array}$ & Chillies and Mirch (Hindi) & Fruit & Capsaicin and Capsicin & - & - \\
\hline 13. & $\begin{array}{l}\text { Cerbara odollum } \\
\text { (Apocynaceae) }\end{array}$ & Dabur, pilikirbir (Hindi) & Fruit and seed & $\begin{array}{l}\text { Cerberin, Cerberoside, } \\
\text { Odollin, Odolotoxin, } \\
\text { Thevetin and Cerapain }\end{array}$ & Kernel of one fruit & $\begin{array}{l}1 \text { - } 2 \text { days or } \\
\text { more }\end{array}$ \\
\hline 14. & $\begin{array}{l}\text { Cerbera thevetia } \\
\text { ( Apocynaceae) }\end{array}$ & $\begin{array}{l}\text { Yellow oleander and Pila } \\
\text { kaner }\end{array}$ & $\begin{array}{l}\text { All parts especially } \\
\text { leaves \& fruits }\end{array}$ & $\begin{array}{l}\text { Thevetin, Thevetoxin, } \\
\text { Nerifolin, Peruvoside, } \\
\text { Ruvoside and Cerberin }\end{array}$ & $\begin{array}{l}8-10 \text { seeds, } 15-20 \mathrm{~g} \text { of } \\
\text { root, } 5 \text { to } 10 \text { leaves }\end{array}$ & $\begin{array}{l}\text { Depend upon } \\
\text { Quantity }\end{array}$ \\
\hline 15. & $\begin{array}{l}\text { Cinchona officinalis } \\
\text { (Rubiaceae) }\end{array}$ & Cinchona & Bark & $\begin{array}{l}\text { Quinine, Cinchonine } \\
\text { and Cinchonidine }\end{array}$ & $8-10 \mathrm{~g}$ & $2 \mathrm{~h}$ to 2 days \\
\hline 16. & $\begin{array}{l}\text { Citrullus colocynthis } \\
\text { (Cucurbitaceae) }\end{array}$ & $\begin{array}{l}\text { Indian wild gourd or bitter } \\
\text { apple, bitter cucumber }\end{array}$ & $\begin{array}{l}\text { Fruit, Root and } \\
\text { dried pulp }\end{array}$ & Colocynthin & 1 - 2 gramColocynthin & $24 \mathrm{~h}$ to $2-3$ days \\
\hline 17. & $\begin{array}{l}\text { Cleistanthus collinus } \\
\text { (Euphorbiaceae) }\end{array}$ & - & Leaves and Bark & Cleistanthin & $0.5 \mathrm{mg} / \mathrm{kg}$ (animals) & - \\
\hline 18. & $\begin{array}{l}\text { Colchicum autumnale } \\
\text { (Colchicaceae) }\end{array}$ & Meadow saffron & & Colchicine & $\begin{array}{l}\text { Similar to arsenic } \\
\text { poisoning }\end{array}$ & $\begin{array}{l}\text { Similar to } \\
\text { arsenic } \\
\text { poisoning }\end{array}$ \\
\hline 19. & $\begin{array}{l}\text { Conium maculatum } \\
\text { (Apiaceae) }\end{array}$ & Poison hemlock & All parts & $\begin{array}{l}\text { Coniine and } \\
\text { Methyl Coniine }\end{array}$ & $1 \mathrm{~cm}$ piece of plant & - \\
\hline 20. & $\begin{array}{l}\text { Crotolaria spectabilis } \\
\text { ( Leguminosae) }\end{array}$ & Jhunjhunia (Hindi) & & Mono-crotaline & 65 mg/kg (chicken) & - \\
\hline 21. & $\begin{array}{l}\text { Croton tiglium } \\
\text { (Euphorbiaceae) }\end{array}$ & $\begin{array}{l}\text { Croton oil seed and } \\
\text { Jamal-gota (Hindi) }\end{array}$ & Seed and oil & $\begin{array}{l}\text { Crotin-a toxal-bumine, } \\
\text { Tiglinic acids, Crotonic acid and } \\
\text { Crotonoside }\end{array}$ & $\begin{array}{l}4 \text { - } 6 \text { seeds, } \\
1-2 \mathrm{ml} \mathrm{oil}\end{array}$ & $6 \mathrm{~h}$ to 3 days \\
\hline 22. & $\begin{array}{l}\text { Cytissus scoparius } \\
\text { (Leguminosae) }\end{array}$ & Yellow broom & $\begin{array}{l}\text { Seed, leaves and } \\
\text { twigs }\end{array}$ & Cytisine and Sparteine & - & - \\
\hline 23. & $\begin{array}{l}\text { Datura fastuosa } \\
\text { (Solanaceae) }\end{array}$ & $\begin{array}{l}\text { Thorn apple and datura } \\
\text { (Hindi) }\end{array}$ & $\begin{array}{l}\text { All parts especially } \\
\text { seeds and fruit }\end{array}$ & $\begin{array}{l}\text { Atropine, Hyoscyamine, Hyscine } \\
\text { and Dutarin }\end{array}$ & 0.6 - 1 gram & $24 \mathrm{~h}$ \\
\hline 24. & $\begin{array}{l}\text { Dieffenbachia sp. } \\
\text { (Araceae) }\end{array}$ & $\begin{array}{l}\text { Dieffenbachia, } \\
\text { dumbcane }\end{array}$ & All parts & $\begin{array}{l}\text { Cyanogenic Glycosides and } \\
\text { Calcium oxalate }\end{array}$ & - & - \\
\hline 25. & $\begin{array}{l}\text { Digitalis purpurea } \\
\text { (Terophularaceae) }\end{array}$ & Fox glove & $\begin{array}{l}\text { Roots, leaves and } \\
\text { seeds }\end{array}$ & $\begin{array}{l}\text { Digitoxin, Digitalin } \\
\text { Digitalein and Digitonin }\end{array}$ & $\begin{array}{l}15-30 \text { mg (Digitalin) } \\
4 \text { mg (Digitoxin) }\end{array}$ & $1 \mathrm{~h}$ to $24 \mathrm{~h}$ \\
\hline 26. & $\begin{array}{l}\text { Dioscorea hispida } \\
\text { (Dioscoreaceae) }\end{array}$ & Karukandu (Hindi) & Tubers & $\begin{array}{l}\text { Spiro Alkaloid } \\
\text { Dioscorine }\end{array}$ & $120 \mathrm{mg} / \mathrm{kg}$ on mice & \\
\hline
\end{tabular}


Citation: Khajja BS, Sharma M, Singh R, Mathur GK (2011) Forensic Study of Indian Toxicological Plants as Botanical Weapon (BW): A Review. J Environment Analytic Toxicol 1:112. doi:10.4172/2161-0525.1000112

Page 4 of 5

\begin{tabular}{|c|c|c|c|c|c|c|}
\hline 27. & $\begin{array}{l}\text { Erythroxylum coca } \\
\text { (Linaceae) }\end{array}$ & Coke, snow & Leaves & $\begin{array}{l}\text { Cocaine, Procaine, } \\
\text { Butacaine and } \\
\text { Dibucaine }\end{array}$ & 1 - 1.5 g Cocaine (Oral) & $15 \mathrm{~min}$ to $10 \mathrm{~h}$ \\
\hline 28. & $\begin{array}{l}\text { Euphorbia helioscopia } \\
\text { (Euphorbiaceae) }\end{array}$ & Sun spurge & Milky latex & $\begin{array}{l}\text { Non-Haemolytic Saponin and } \\
\text { Phasin }\end{array}$ & - & - \\
\hline 29. & $\begin{array}{l}\text { Gloriosa superba } \\
\text { (Liliaceae) }\end{array}$ & $\begin{array}{l}\text { Superb lily, Flame lily and } \\
\text { Kalihari (Hindi) }\end{array}$ & Tubers and roots & $\begin{array}{l}\text { Colchicine, Superbine, } \\
\text { Gloriosine and } \\
\text { Glucosine }\end{array}$ & - & - \\
\hline 30. & $\begin{array}{l}\text { Gossypium sp. } \\
\text { (Malvaceae) }\end{array}$ & Kapas (Hindi) & Seed oil & Gossypol & $2.57 \mathrm{~g} / \mathrm{kg}$ (Rat) & \\
\hline 31. & $\begin{array}{l}\text { Hyoscyamus niger } \\
\text { (Solanaceae) }\end{array}$ & $\begin{array}{l}\text { Henbane and Ajwayan } \\
\text { (Hindi) }\end{array}$ & All parts & $\begin{array}{l}\text { Atropine, Hyoscine and } \\
\text { Hyscyamine }\end{array}$ & 125mg (hyoscyamine) & $24 \mathrm{~h}$ \\
\hline 32. & $\begin{array}{l}\text { Jatropa multifida } \\
\text { (Euphorbiaceae) }\end{array}$ & Bherenda (Hindi) & foliage and fruits & Curcin & - & - \\
\hline 33. & $\begin{array}{l}\text { Lantana camara } \\
\text { (Verbenaceae) }\end{array}$ & Lantana, bunch berry & $\begin{array}{l}\text { Entire plant, } \\
\text { especially the } \\
\text { berries }\end{array}$ & $\begin{array}{l}\text { Lantanine, Lancamarone and } \\
\text { Lantadenes A,B }\end{array}$ & - & - \\
\hline 34. & $\begin{array}{l}\text { Lasiosiphon eriocephalus } \\
\text { ( Thymeleaceae) }\end{array}$ & $\begin{array}{l}\text { Rameetha } \\
\text { (Hindi) }\end{array}$ & $\begin{array}{l}\text { Stem, leaves and } \\
\text { bark }\end{array}$ & $\begin{array}{l}\text { Lasioside and } \\
\text { Lasiocephatin }\end{array}$ & $0.5 \mathrm{mg} / \mathrm{kg}$ roots (cats) & - \\
\hline 35. & $\begin{array}{l}\text { Lathyrus sativus } \\
\text { (Fabaceae) }\end{array}$ & Gross pea, Khesari (Hindi) & Seeds & $\begin{array}{l}\beta \text {-Aminopropionitrile, } \\
\beta \text {-Cyanoalanine,2,4-diamino } \\
\text { Butyric acid, Selenium and } \\
\text { 3-N-oxalyl-2,3-Di- } \\
\text { aminopropinoic acid }\end{array}$ & - & - \\
\hline 36. & $\begin{array}{l}\text { Lobelia nicotiancefolia } \\
\text { (Campanulaceae) }\end{array}$ & $\begin{array}{l}\text { Indian lobelia and deonal } \\
\text { (Hindi) }\end{array}$ & All parts & Lobeline & $\begin{array}{l}10 \text { mg (Lobeline), } \\
3.75 \text { gram(leaves) }\end{array}$ & 30 min to a day \\
\hline 37. & $\begin{array}{l}\text { Manihot esculenta } \\
\text { (Euphorbiaceae) }\end{array}$ & Sakarkanda (Hindi) & Tubers & Cyanogenic Glycoside & - & - \\
\hline 38. & $\begin{array}{l}\text { Manihot utilissima } \\
\text { (Euphorbiaceae) }\end{array}$ & $\begin{array}{l}\text { Cassava, } \\
\text { tapioca }\end{array}$ & Root and leaves & $\begin{array}{l}\text { Linamarin- a Cyanogenic } \\
\text { Glycoside }\end{array}$ & $\begin{array}{l}300 \text { gram(adult) } \\
125 \mathrm{~g} \text { (child) } \\
\text { fresh root }\end{array}$ & - \\
\hline 39. & $\begin{array}{l}\text { Mucuna prurita } \\
\text { (Leguminosae) }\end{array}$ & $\begin{array}{l}\text { Velvet bean, Konch } \\
\text { (Hindi) }\end{array}$ & Seeds & $\begin{array}{l}\text { Mucunain, } \\
\text { Serotonin }\end{array}$ & - & - \\
\hline 40. & $\begin{array}{l}\text { Myristica fragrans } \\
\text { ( Myristicaceae) }\end{array}$ & $\begin{array}{l}\text { Nutmeg, } \\
\text { Mace tree }\end{array}$ & Seeds & Myristicin and Elemicin & $\begin{array}{l}1 \text { - } 3 \text { nutmegs (adult) } \\
2 \text { nutmegs (child) }\end{array}$ & - \\
\hline 41. & $\begin{array}{l}\text { Nerium odorum } \\
\text { (Apocynaceae) }\end{array}$ & $\begin{array}{l}\text { White oleander and Kaner } \\
\text { (Hindi) }\end{array}$ & All parts & $\begin{array}{l}\text { Neriodorin, Neriodorein and } \\
\text { Karabin }\end{array}$ & $\begin{array}{l}15-20 \text { g root } \\
/ 24-36 \text { hours }\end{array}$ & \\
\hline 42. & $\begin{array}{l}\text { Nicotiana tabacum } \\
\text { (Solanaceae) }\end{array}$ & $\begin{array}{l}\text { Tobacco and tambaku } \\
\text { (Hindi) }\end{array}$ & $\begin{array}{l}\text { All parts except ripe } \\
\text { seeds }\end{array}$ & Nicotine & $\begin{array}{l}60-100 \mathrm{mg} \text { nicotine or } 2 \\
\text { gramtobacco }\end{array}$ & $5-15 \min$ \\
\hline 43. & $\begin{array}{l}\text { Ochrocarpus longifolius } \\
\text { (Gutiferae) }\end{array}$ & Naag kesar & All parts & $\begin{array}{l}\text { Surangin A } \\
\text { and Surangin B }\end{array}$ & $\begin{array}{l}\mathrm{LD}-9 \mathrm{mg} / \mathrm{kg} \\
\text { surangin } \mathrm{A} \text { and } \\
1 \mathrm{mg} / \mathrm{kg} \text { surangin } \mathrm{B} \\
\text { (Cats) }\end{array}$ & - \\
\hline 44. & $\begin{array}{l}\text { Papaver somniferum } \\
\text { (Papavaraceae) }\end{array}$ & $\begin{array}{l}\text { Opium poppy and afim } \\
\text { (Hindi) }\end{array}$ & $\begin{array}{l}\text { Ripe and dried } \\
\text { capsules, petals } \\
\text { and seeds }\end{array}$ & $\begin{array}{l}\text { Morphine, } \\
\text { Narcotine, Codeine } \\
\text { and Thebaine }\end{array}$ & $\begin{array}{l}2 \text { gram(opium), } \\
0.2 \text { gram(morphine) } \\
\text { and } 0.5 \text { gram(codeine) }\end{array}$ & 2 to $6 \mathrm{~h}$ \\
\hline 45. & $\begin{array}{l}\text { Parthenium } \\
\text { hysterophorus } \\
\text { ( Compositae) }\end{array}$ & Carrot grass & Leaves and seeds & Parthenin & - & - \\
\hline 46. & $\begin{array}{l}\text { Pegnum hermala } \\
\text { (Zygophyllaceae) }\end{array}$ & Wild rue & All parts & $\begin{array}{l}\text { Harmaline, Harmine, Harmane, } \\
\text { Harmalol, } \\
\text { Asicine, } \\
\text { Vasicinone }\end{array}$ & $\begin{array}{l}\text { Harmaline-120 mg/kg } \\
\text { (rat), } \\
\text { Harman-200 mg/kg } \\
\text { (rabbit) }\end{array}$ & - \\
\hline 47. & $\begin{array}{l}\text { Plumbago zeylanica } \\
\text { (Plumbaginaceae) }\end{array}$ & $\begin{array}{l}\text { Ceylon leadwort and chitra } \\
\text { (Hindi) }\end{array}$ & Root & Plumbagin & $5 \mathrm{gm}$ & Few days \\
\hline 48. & $\begin{array}{l}\text { Prunus amygdalus } \\
\text { ( Rosaceae ) }\end{array}$ & $\begin{array}{l}\text { Almond, } \\
\text { Baadam (Hindi) }\end{array}$ & Almond & Amygdalin & $\begin{array}{l}20 \text { almonds (adult) } \\
10 \text { almonds (Child) }\end{array}$ & \\
\hline 49. & $\begin{array}{l}\text { Rauvolfia serpentina } \\
\text { (Apocynaceae) }\end{array}$ & Chandrabhaga (Hindi) & Roots & $\begin{array}{l}\text { Reserpine, Reserpidine, } \\
\text { Reserpinine and } \\
\text { Serpentine }\end{array}$ & - & - \\
\hline 50. & $\begin{array}{l}\text { Ricinus communis } \\
\text { (Euphorbiaceae) }\end{array}$ & $\begin{array}{l}\text { Castor bean, Erandi } \\
\text { (Hindi) }\end{array}$ & $\begin{array}{l}\text { Entire plant } \\
\text { especially seeds }\end{array}$ & Recine and Recinine & $\begin{array}{l}6 \mathrm{mg} \text { of ricin } \\
\text { (10 seeds) }\end{array}$ & $\begin{array}{l}2 \text { to several } \\
\text { days }\end{array}$ \\
\hline 51. & $\begin{array}{l}\text { Semecarpus anacardium } \\
\text { (Anacardiaceae) }\end{array}$ & $\begin{array}{l}\text { Marking nut } \\
\text { And bhilawa (Hindi) }\end{array}$ & Juice & $\begin{array}{l}\text { Semecarpol and } \\
\text { Bhilawanol }\end{array}$ & $5-10 \mathrm{gm}$ & $12-24 h$ \\
\hline 52. & $\begin{array}{l}\text { Solanum nigrum } \\
\text { ( Solanaceae) }\end{array}$ & Black nightshade & Immature berries & Solanine and Steroids & - & - \\
\hline 53. & $\begin{array}{l}\text { Strychnos nux-vomica } \\
\text { (Loganiaceae) }\end{array}$ & $\begin{array}{l}\text { Poison nut and Kuchila } \\
\text { (Hindi) }\end{array}$ & $\begin{array}{l}\text { All parts especially } \\
\text { seeds }\end{array}$ & Strychnine, Brucine and Vomicine & $\begin{array}{l}15-20 \mathrm{mg} / \mathrm{kg} \\
(1 \text { seed }- \text { Oral) }\end{array}$ & $1-2 h$ \\
\hline 54. & $\begin{array}{l}\text { Thevetia peruviana } \\
\text { (Apocynaceae) }\end{array}$ & $\begin{array}{l}\text { Suicide tree and Pila } \\
\text { kaner (Hindi) }\end{array}$ & $\begin{array}{l}\text { Seeds and milky } \\
\text { juice }\end{array}$ & $\begin{array}{l}\text { Thevetin A and } \\
\text { Thevetin B }\end{array}$ & $\begin{array}{l}\text { Kernel of } 01 \text { fruit, } \\
2 \text { leaves (child) }\end{array}$ & - \\
\hline
\end{tabular}

Table 1: Review of the toxic/poisonous Indian plants. 
Citation: Khajja BS, Sharma M, Singh R, Mathur GK (2011) Forensic Study of Indian Toxicological Plants as Botanical Weapon (BW): A Review. J Environment Analytic Toxicol 1:112. doi:10.4172/2161-0525.1000112

Page 5 of 5

There are some plants like Cerbera odollum, Cleistanthus collinus, Strychnos nux -vomica,Abrus precatorius, Colchicum autumnale, and Datura metel are very toxic plants and used for homicidal and suicidal purposes. Almost no other plant has such a history of crime as datura and the seeds are favorite poisons used for this purpose. Colchicum autumnale poisoning has been compared with arsenic poisoning. Those plants which are allergens or less poisonous, are used in burglary purpose in buses/trains Mucuna prurita is most useful plants for burglary purpose. Sometimes accidental poisoning by poisonous plants has also been observed in tribal area of south Rajasthan.

To this day, some writers continue to talk about ricin as a potential bioterrorism weapon insisting that Iraq was producing ricin but completely ignoring the evidence that this production was small scale, terminated in the mid 2000s and was for use in assassination [20-21].

Significance of this review on such plants helps to forensic investigators in solving the crime and searching the poisoning- plantmaterials on crime spot. On crime spot forensic connoisseur can get evidence related to suicidal, accidental or homicidal poisoning by poisonous plants like parts of plants, spot of crime like forest/garden, symptoms of poisoning can help to direct the investigation of right path. On the basis of plant origin toxicity forensic team can tell whether it is suicide, homicide or accident.

\section{References}

1. Narayan Reddy K S, Medical Jurisprudence and Toxicology (law practice \& procedure) ALT Publications, Hyderabad (2005).

2. Parikh $\mathrm{CK}$, Textbook of Medical Jurisprudence and Toxicology, CBS publisher, Mumbai $6^{\text {th }}$ Ed. (2005).

3. Greval SDS, Lyon's Medical Medical Jurisprudence for India $10^{\text {th }}$ Ed. DLH (1953).

4. Viswanathan N, Joshi B S (1983) Toxic constituents on some Indian Plants. Current Science $52: 1-8$

5. Subrahmanyam BV, Modi's Medical Jurisprudence and Toxicology, $22^{\text {nd }}$ Ed. Butterworths, India (1999).

6. Chopra R N, Badhwar R L, Ghosh S (1949) Poisonous Plants of India Vol.1 (ICAR, New Delhi)
7. Boesche, Roger (2003) "Kautilya's Arthasastra on War and Diplomacy in Ancient India". The Journal of Military History 67 : 9-37. ISSN 0899-3718.

8. Qureshi JM, Bano S, Mohammad T, Khan MA (2001) Medicinal potential of poisonous plants of tehsil Kahuta from district Rawalpindi,Pakistan.Pakistan Journal of Biological Sciences. 4 :331-332.

9. Ballantyne B, Mars T C, Turner P (1995) Fundamentals of toxicology, Genera and Applied Toxicology, McMillan Press.

10. Singh D, Jit I, Tyagi S (1999) Changing trends in acute poisoning in Chandigarh zone: A 25 year autopsy experience from a tertiary care hospital in Northern India. Amer J Forensic Med Pathol 20: 203-210.

11. Murari A, Sharma GK (2002) A comparative study of poi-soning case autopsied in LHMC New Delhi and JIP-MER Pondicherry. J Forensic Med Toxicol 19: 19-21.

12. Siwach SB, Gupta A (1995) The profile of acute poisonings in Haryana: Rohtak study. J Assoc Physicians India 43: 756-759.

13. Singh LR, Momonchand A and Singh PI 2001. Pattern of accidental poisoning in children. J Indian Acad Forensic Med. 23: 69-71.

14. Dash SK, Mohanty MK and Mohanty S (2005) Sociodemo-graphic profile of poisoning cases. J Indian Acad Fo-rensic Medicine 27: 133-138.

15. Caius J F (2003) The Medicinal and Poisonous Plants of India, (Scientific Publisher, Jodhpur).

16. Burkill, HN (1985) The useful plants of West Africa (tropical) Ed. 2 Vol. I. Families A.D. Royal Botanical Garden, Kew.

16b) Neuwinger, HD (2004) Review of plants used for poison fishing in tropical Africa. Toxicon. 44: 417-430.

17. Caius JF (2003) The Medicinal and Poisonous Plants of India, Scientific Publishers, Jodhpur.

18. Windholz M (1983) The Merck Index: an encyclopedia of chemicals, drugs, and biologicals, Rahway, New Jersey, Merck and Co., Inc. 10th ed.

19. Dogra T D, Lt Col Rudra A (2005) Lyon's Medical Jurisprudence and Toxicology Delhi Law House Publishers, New Delhi, $11^{\text {th }}$ Ed.

20. Is that cat dead? And other questions about poison plants by John Robertson Book Guild Publishing (2010) ISBN-9781846244254.

21. Miller Coyle H, Ladd C, Palmbach T, Lee HC (2001) The Green Revolution: botanical contributions to forensics and drug enforcement. Croat Med $\mathrm{J} 42$ 340-345. 